\title{
Efeito de Técnicas Fisioterápicas sobre os Desconfortos Músculo-esqueléticos da Gestação
}

\author{
Effect of Physiotherapeutic Techniques on Musculoskeletal Discomforts in Pregnancy
}

Marta Helena Souza De Conti ${ }^{1,2}$, Iracema de Mattos Paranhos Calderon ${ }^{1}$, Elenice Bertanha Consonni ${ }^{1}$, Tânia Terezinha Sculler Prevedel ${ }^{1}$, Ivete Dalbem ${ }^{3}$, Marilza Vieira Cunha Rudge ${ }^{1}$

\section{RESUMO}

Objetivo: estudar os efeitos de técnicas fisioterápicas sobre os desconfortos músculoesqueléticos na gestação.

Métodos: estudo coorte prospectivo, com 71 gestantes nuliparas, de baixo risco, distribuidas conforme a participação (estudo; $n=38$ ) ou não (controle; $n=33$ ). O Programa Multidisciplinar de Preparo para o Parto e Maternidade constou de 10 encontros (da $18^{a}$ à $38^{a}$ semana), com atividades educativas, fisioterápicas e de interação. Compararam-se, por questionário específico, no inicio e final do programa, a ocorrência (presença ou ausência), as características (local, tipo, intensidade, freqüência e duração) e a evolução dos desconfortos músculoesqueléticos. As médias aferidas na avaliação inicial foram comparadas pela análise de variância (ANOVA) seguida pelo teste do F. Para o estudo entre as proporções, verificadas no início e no final do programa, utilizou-se o teste do $\chi^{2}$. A significância estatística foi definida pelo limite de $5 \%(p<0,05)$.

Resultados: no inicio do programa, 63,6\% das gestantes do grupo controle e 84,2\% do Estudo relataram sintomas músculo-esqueléticos $(p=0,05)$, caracterizados por dor na região lombossacra. No grupo controle predominaram a intensidade leve (18,2\%) e grave (18,4\%), e no grupo estudo, a grave (36,8\%) e isolada ou associada (31,6\%). No final, o grupo controle tinha sintomas de intensidade grave $(60,6 \%)$, com freqüência diária $(42,4 \%)$ e duração maior que três horas $(69,7 \%)(p<0,05)$. O grupo estudo referia intensidade leve (57,9\%) e freqüência quinzenal $(50,0 \%)$ com duração máxima de uma hora $(55,3 \%)(p<0,05)$. A evolução dos sintomas foi diferenciada, confirmando-se piora em $63,6 \%$ das gestantes do Controle $e$ melhora em 65,8\% das participantes do Programa ( $p<0,05)$.

Conclusões: as técnicas fisioterápicas do Programa Multidisciplinar de Preparo para o Parto e Maternidade se relacionaram à diminuição da intensidade, freqüência e duração e à melhor evolução dos desconfortos músculo-esqueléticos na gestação.

PALAVRAS-CHAVE: Gravidez normal. Desconforto músculo-esquelético. Programas de preparo à maternidade.

${ }^{1}$ Disciplina de Obstetrícia, Faculdade de Medicina de Botucatu - UNESP

${ }^{2}$ Faculdade de Fisioterapia, Universidade do Sagrado Coração - Bauru

${ }^{3}$ Departamento de Medicina Preventiva, Faculdade de Medicina de Botucatu - UNESP

Correspondência:

Marta Helena Souza De Conti

Rua Jacinto Ghuiraldeli O-678 - Jardim Alvorada

17280-000 - Pederneira - SP

e-mail: madeconti@yahoo.com.br

\section{Introdução}

Nas últimas décadas, o enfoque da assistência ao pré-natal, ao parto e à maternidade em si tem sido bem diferenciado. Os programas multidisciplinares de preparação para o parto, caracterizados pelo desenvolvimento de métodos educativos, atenção psicológica e preparo físico específico, estão se tornando comuns e cada vez 
mais procurados pela maioria das gestantes ${ }^{1-3}, \mathrm{com}$ o objetivo de assegurar o controle sobre seu corpo e sua gestação, introduzindo-as na prática de exercícios que resultam em equilíbrio físico e psíquico, com conseqüente sensação de bem-estar ${ }^{1,4}$.

Tal prática vem sendo cada vez mais discutida e, de modo especial, as possiveis repercussões maternas e fetais. Os beneficios foram atribuídos à diminuição dos sintomas de desconfortos da gravide $z^{5}$, ao controle da ansiedade ${ }^{4}$ e depressão ${ }^{6}$, ao menor tempo de evolução do trabalho de parto ${ }^{4,7}$ e ao menor índice de indicação de parto cesárea ${ }^{4,6}$. Entretanto, alguns resultados desfavoráveis foram observados na aplicação de exercícios intensos, entre eles, a ocorrência de prematuridade e de baixo peso ${ }^{1}$.

Sob o ponto de vista fisioterápico, a maioria destes programas foi prescrita para melhorar a força e as condições das estruturas de sustentação do $\mathrm{corpo}^{2}$. Os exercícios aplicados (cinesioterapia - terapia por movimento - recurso amplamente difundido entre fisioterapeutas) ${ }^{8}$ ajudam na manutenção da postura da coluna vertebral, promovem adaptações biomecânicas mais eficientes e atuam na prevenção ou controle do estresse e das dores referidas nos segmentos lombar e pélvico ${ }^{9}$.

O Colégio Americano de Ginecologia e Obstetrícia apostando nos beneficios maternos e fetais, definiu algumas recomendações de interesse na prescrição de programas de exercícios na gravidez. Independentemente do tipo, as intensidades leve ou moderada são as mais adequadas, com freqüência mínima de três vezes por semana, mantida regularmente. Devem ser evitadas as atividades intensas; é importante respeitar o limite de $140 \mathrm{bpm}$ para a freqüência cardiaca materna e de $38^{\circ} \mathrm{C}$ para a temperatura ambiente ${ }^{1,2,10,11}$. Os exercícios de solo devem ser realizados em superficies firmes e regulares $^{1,2}$ e de maneira geral, assegurar melhor controle da percepção corporal, da auto-estima, do humor e da ansiedade, proporcionando sensação de bem-estar físico e psicológico ${ }^{4,10,11}$.

O bem-estar físico relaciona-se à ausência ou a mínimos graus de doença, incapacidade ou desconfortos, em especial, relacionados ao sistema músculo-esquelético ${ }^{12}$. O desconforto músculo-esquelético seria, mais comumente, definido por fenômeno de percepção física desagradável relacionado a fadiga aguda e sobrecarga fisica. Sua identificação pode ser feita por relatos objetivos (questionários específicos) a partir de avaliações da ocorrência (presença ou ausência) e das características dos sintomas, tais como, o local, o tipo, a intensidade, a freqüência e a duração do desconforto ${ }^{13}$. Ao longo do tempo os métodos para avaliar os desconfortos músculo-esqueléticos percebidos foram adaptados por diversos investigadores que o transformaram em questionário para localizar e identificar os sintomas ${ }^{12,13}$. Mais intensos e freqüentes no final da gestação, principalmente na região abdominal baixa, os desconfortos músculo-esqueléticos podem se manifestar por sensação de peso ou "repuxos" na região pélvica ou sacroilíaca $^{14}$. Estima-se que 50 a $70 \%$ das gestantes normais são acometidas por dores nas costas e que o risco pode ser aumentado naquelas que já sofriam desses sintomas antes da gravide $z^{6}$.

Alguns protocolos já foram testados, enfocando a cinesioterapia com alongamento de grupos musculares específicos - peitorais, adutores da coxa, paravertebrais lombares, quadrado lombar e musculatura posterior dos membros inferiores - e o fortalecimento de outros músculos - perineais e abdutores das coxas, sujeitos à sobrecarga mecânica e funcional durante a gestação e parto ${ }^{2,15,16}$. Esses exercícios, envolvendo grupos musculares estressados, aumentaram a capacidade funcional e facilitaram a compensação muscular, reduzindo os sintomas de dor e/ou de desconfortos na gravidez e puerpério ${ }^{9,15}$.

A partir dos fundamentos relacionados aos benefícios da fisioterapia e às adaptações osteoarticulares maternas à gestação, é válido incluir a cinesioterapia em programas de assistência multidisciplinar à maternidade. De modo especial, esta técnica fisioterápica, realizada em solo, deveria ser investigada por seus efeitos benéficos sobre os desconfortos músculo-esqueléticos maternos, sem prejuízo fetal, além de supostamente prática e de baixo custo. Ao ser utilizada com esse enfoque específico, a cinesioterapia no solo deverá proporcionar, no minimo, melhor qualidade de vida durante este período.

Este trabalho teve como objetivo geral estudar os efeitos de técnicas fisioterápicas aplicadas no Programa Multidisciplinar de Preparo para o parto e Maternidade (PMPMa) sobre os desconfortos músculo-esqueléticos da gestação. Foram objetivos específicos comparar a ocorrência, as características (relativas ao local, tipo, intensidade, freqüência e duração) e a evolução dos desconfortos músculo-esqueléticos entre gestantes participantes e não participantes do PMPMa, no início e final do programa.

\section{Métodos}

Esta pesquisa foi realizada no Serviço de Obstetrícia-assistência ao pré-natal, parto e puerpério, da Faculdade de Medicina de Botucatu (FMB- 
UNESP), no período de setembro de 1999 a maio de 2001. Trata-se de ensaio clínico, sobre os resultados de técnicas fisioterápicas desenvolvidas no PMPMa. Foram constituídos dois grupos de pacientes, de acordo com a participação (grupo estudo- GE) ou não no programa (grupo controle - GC).

Participaram do estudo gestantes acompanhadas no pré-natal especializado de adolescentes e primigestas FMB-UNESP, que preencheram os seguintes critérios de inclusão: nuliparidade, ausência de doença clínica ou obstétrica, gestação única, idade gestacional entre a $18^{\mathrm{a}}$ e a $22^{\mathrm{a}}$ semana e o desejo de participar de um programa de preparo para o parto.

A falta de espaço físico, material e de pessoal impossibilitou a seleção aleatória (randomização) e foram formados cinco subgrupos do GE (com 6 a 8 grávidas cada), de forma subseqüente, até completar 40 participantes. As gestantes do GC foram selecionadas após o preenchimento de cada subgrupo do GE e o recrutamento também se estendeu até atingir 40 gestantes. O GC não sofreu nenhum outro tipo de intervenção além da assistência pré-natal de rotina do Serviço. Todas as grávidas, independente do grupo, assinaram o termo de consentimento livre e esclarecido, confirmando o aceite em participar deste estudo.

Consideraram-se como critérios de descontinuidade a desistência da assistência prénatal no Serviço, o parto fora da Maternidade da FMB - Unesp e a não-adesão ao PMPMa, definida pela ausência em três reuniões, consecutivas ou não.

O PMPMa foi aplicado apenas para as gestantes do GE, nas manhãs que antecederam as consultas do pré-natal. Constituiu de 10 encontros, com freqüência quinzenal nos seis primeiros e semanal nos quatro últimos. Cada encontro teve duração de três horas e foi composto de três atividades básicas: educativa, fisioterápica e de interação. Os encontros iniciaram-se com a atividade educativa envolvendo todas as gestantes do subgrupo, com duração aproximada de $50 \mathrm{mi}-$ nutos. Após um intervalo de 15 a 20 minutos, quando foi oferecido lanche, metade das gestantes participou alternadamente das outras atividades de interação e fisioterápica, com duração média de 50 minutos cada.

A atividade educativa ofereceu informações pertinentes à gravidez, parto, puerpério e cuidados com o recém-nascido. Os temas, previamente escolhidos, foram apresentados pelos profissionais de saúde do Serviço e discutidos de maneira bastante informal com as gestantes. Foi incluída uma visita à maternidade, onde as participantes conheceram a Enfermaria de Obstetrícia, o alojamento conjunto, as salas de pré-parto e parto e o berçário.
A atividade de interação foi coordenada pela psicóloga e representou espaço de discussão sobre as experiências com a gestação, as vivências emocionais envolvidas na situação de ter um filho e o impacto da gravidez no contexto familiar.

A atividade fisioterápica foi coordenada pela fisioterapeuta da equipe, que aplicou técnicas fisioterápicas, padronizadas de acordo com Polden e Mantle ${ }^{2}$ e desenvolvidas na seguinte ordem: treino respiratório - as gestantes foram treinadas a adotar o padrão respiratório 1:1 (inspiração nasal profunda diafragmática seguida de expiração oral frenolabial $)^{17}$, específicas para os intervalos entre as contrações do trabalho de parto. Após a $36^{a}$ semana, além da prática respiratória, elas foram treinadas para a manobra de Valsalva, a ser aplicada no período expulsivo do parto. O treino respiratório teve duração máxima de oito minutos, com pequenas pausas para descanso a cada três ou quatro movimentos respiratórios. Iniciava-se com as gestantes em decúbito lateral esquerdo e, posteriormente, era alterada para a posição supina.

Para cinesioterapia, foram desenvolvidas seqüências de exercícios de alongamento e fortalecimento muscular de intensidade leve ${ }^{1}$, adequadas para a idade gestacional. As seqüências eram iniciadas por exercícios metabólicos ${ }^{2}$ nos membros superiores - fechamento e abertura dos dedos das mãos - e nos membros inferiores - dorsoflexão e circuncisão do tornozelo ${ }^{2}$-,e finalizadas com a série de Williams ${ }^{18}$, adaptada para a gestação. Essa série compreende alongamento dos músculos póstero-inferiores do tronco, quadrado lombar, glúteos, ísquio-tibiais e músculos adutores da coxa e fortalecimento dos músculos abdutores da coxa e do assoalho pélvico, principalmente os do períneo. Nesta seqüência de exercícios foram também aplicados exercícios para o alongamento dos músculos cervicais, peitorais e os das cadeias anterior e posterior dos membros superiores.

Cada sessão de cinesioterapia teve duração média de trinta minutos, com pequenas pausas para descanso, e todos os exercícios foram associados ao padrão respiratório $1: 1$. Durante esta prática a freqüência cardíaca $(\mathrm{FC})$ materna não ultrapassou $140 \mathrm{bpm}^{1}$.

As sessões de orientações posturais, fizeram duração máxima de doze minutos, gestantes eram posicionadas para relaxamento corporal e recebiam informações sobre posturas corretas para dormir - em decúbito lateral esquerdo, com um travesseiro sob a cabeça e outro entre as pernas, suavemente flexionadas; para sentar - os pés apoiados no chão e os segmentos da coluna dorsal e lombar totalmente encostados na cadeira, e para levantar-se da cama-em decúbito lateral, apoiadas nos membros superiores. 
As grávidas foram também treinadas para as atividades de vida diárias, recebendo informações sobre a melhor postura para realizar os afazeres domésticos, entre as quais tronco e membros inferiores semiflexionados para permanecer em pé em frente à pia da cozinha, ao tanque, à mesa de passar roupas e para a limpeza do chão, e para as atividades de higiene e cuidados pessoais - tronco e membros inferiores semiflexionados diante do lavatório do banheiro, flexão e abdução coxofemoral na posição sentada para a colocação de sapatos. Ao término de cada encontro foram orientadas a praticar as atividades fisioterápicas em casa, com freqüência mínima de três vezes por semana.

A participação ou não no programa foi considerada variável independente. Como variáveis de controle foram relacionadas a idade materna, o peso materno inicial (18-20 semanas de gestação), o indice de massa corporal (IMC) - calculado pelo peso pré-gravídico $\left(\mathrm{kg} / \mathrm{m}^{2}\right)$, também no início do Programa (18-20 semanas de gestação ${ }^{19}$, a renda familiar, o grau de escolaridade - categorizado em ensino fundamental (menor ou igual a 8 anos), ensino médio (de 9 a 11) e nivel superior (maior ou igual a 12 anos) ${ }^{20}$ - e a situação conjugal - considerada pela condição de morar ou não com o companheiro.

Foram consideradas variáveis dependentes a ocorrência, as características e a evolução dos desconfortos músculo-esqueléticos durante a gestação. Para a comparação destas variáveis aplicouse o Questionário de Desconforto MúsculoEsquelético Percebido ${ }^{12}$ em dois momentos, entre a $18^{\mathrm{a}}$ e a $22^{\mathrm{a}}$ semana (início) e na $38^{\mathrm{a}}$ semana (final) de gestação. A ocorrência foi considerada pela presença ou ausência de sintomas de desconfortos músculo-esqueléticos. O Questionário de Desconforto Músculo-Esquelético Percebido foi aplicado por ser método freqüentemente utilizado na literatura para avaliar, identificar e localizar os relatos de sintomas em quaisquer indivíduos ${ }^{12,13}$.

As características relativas ao local, tipo, intensidade, freqüência e duração foram analisadas separadamente. O local foi categorizado por regiões, definidas como cérvico-torácica, (segmentos músculo-esqueléticos da região cervical, dos membros superiores e do tórax anterior e posterior) e lombo-sacra (segmentos lombar, sacral e membros inferiores). O tipo foi referido como dor e sensação de peso e/ou parestesia (formigamento e outros). A intensidade foi diferenciada em graus leve (fraca), moderada e grave (forte, intenso ou insuportável). A freqüência foi relacionada como quinzenal (uma a três vezes ao mês e/ou infreqüente), freqüente (uma vez por semana) e diária (quase todo dia e/ou diária). A duração foi definida por intervalos de alguns minutos até 1 hora (maior ou igual a 1 hora), de algumas horas (2-3 horas), de várias horas (maior que 3 horas) e não regulares. A multiplicidade das queixas, relativas a quaisquer das características avaliadas, foi considerada em associação (associadas).

Para a evolução, além da ausência de desconforto (sem sintomas), consideraram-se as formas possiveis de evolução de suas caracteristicas, avaliadas entre o início e o final do programa, assim definidas: inalterada, nos casos que não apresentaram nem melhora e nem piora do quadro inicial, em qualquer característica; piora, naqueles casos em que se observou acentuação de qualquer uma das características, e melhora, para os casos que apresentaram regressão de pelo menos uma das características avaliadas.

No estudo estatístico, as médias aferidas na avaliação inicial foram comparadas pela análise de variância (ANOVA) seguida pelo teste do $\mathrm{F}^{21}$. Para o estudo entre as proporções, verificadas no início e no final do programa, utilizou-se o teste do $\chi^{2}$, com as devidas correções, quando necessá$\operatorname{rias}^{21}$. A confirmação de significância estatística foi definida pelo limite de $5 \%(p<0,05)$.

Este projeto foi aprovado pelo Comitê de Ética em Pesquisa da FFMB-UNESP.

\section{Resultados}

Aceitaram participar deste estudo 80 gestantes, distribuídas entre GC e GE. No GE foram desconsideradas duas grávidas, por não-adesão ao PMPMa. Das 40 gestantes do GC, sete foram desconsideradas na análise dos resultados: uma por diagnóstico tardio de gemelaridade, três por parto fora do serviço e três por desistência da assistência pré-natal. Desta forma, foram comparados os resultados de 38 gestantes do GE e 33 do GC.

As características pessoais, avaliadas entre 18 e 20 semanas de gestação, antes do início do PMPMa, não foram diferentes do ponto de vista estatístico e não diferenciaram os grupos de gestantes. O peso corporal inicial, o IMC, a renda familiar, a situação conjugal e a escolaridade foram estatisticamente semelhantes entre os grupos, à exceção da idade materna. No GC a idade média foi de 20,8 e no GE, de 17,9 anos ( $p<0,05)$.

A ocorrência (ausência ou presença) dos sintomas de desconforto músculo-esqueléticos não foi diferente entre os grupos nos momentos inicial e final. Apesar disso, a análise estatística mostrou tendência $(p=0,05)$ das grávidas de serem mais sintomáticas na avaliação inicial. A evolução destes sintomas foi diferenciada entre os grupos, pela 
maior proporção de grávidas que referiram piora no GC $(63,6$ vs $7,9 \%)$ e melhora no GE $(65,8$ vs $3,1 \%)$ (Tabela 1).

Independentemente do grupo, a maior parte das gestantes relatou predominio do desconforto músculo-esquelético localizado na região lombo-sacra e manifestado por sintomas de dor, tanto no início como no final do programa. Nestas características não foram observadas diferenças significativas.

Tabela 1 - Número (n) e porcentagem (\%) de gestantes classificadas de acordo com a ocorrência (presença ou ausência) no início e final do programa e a evolução dos sintomas de desconfortos músculo-esqueléticos, nos grupos controle e estudo.

\begin{tabular}{|c|c|c|c|c|}
\hline \multirow[b]{2}{*}{ Ocorrência* } & \multicolumn{2}{|c|}{ Controle } & \multicolumn{2}{|c|}{ Estudo } \\
\hline & $\mathrm{n}$ & $\%$ & $\mathrm{n}$ & $\%$ \\
\hline \multicolumn{5}{|l|}{ |nicial* ${ }^{*}$} \\
\hline Ausente & 12 & 36,4 & 6 & 15,8 \\
\hline Presente & 21 & 63,6 & 32 & 84,2 \\
\hline \multicolumn{5}{|l|}{ Final } \\
\hline Ausente & 4 & 12,1 & 9 & 23,7 \\
\hline Presente & 29 & 87,9 & 29 & 76,3 \\
\hline \multicolumn{5}{|l|}{ Evolução } \\
\hline Sem sintomas & 4 & 12,1 & 4 & 10,5 \\
\hline Inalterada & 7 & 21,2 & 6 & 15,8 \\
\hline Piora** & 21 & 63,6 & 3 & 7,9 \\
\hline Melhora** & 1 & 3,1 & 25 & 65,8 \\
\hline
\end{tabular}

${ }^{*} p=0,05$ - diferença não significativa entre os grupos na avaliação inicial.

${ }^{* *} p<0,05$ - diferença significativa entre os grupos na avaliação final.
$\mathrm{Na}$ avaliação inicial as gestantes do GC relatavam, predominantemente, desconfortos de intensidade leve em $18,2 \%$ e grave em $18,4 \%$. Neste mesmo momento, o GE foi caracterizado por intensidades consideradas graves $(36,8 \%)$ e associadas $(31,6 \%)$. No final, a maioria das gestantes do GC $(60,6 \%)$ se queixou de intensidade grave e $23,7 \%$ delas referiam graus associados. No GE, 57,9\% das gestantes relataram desconfortos de intensidade leve, seguidos de ausência de sintoma em $23,7 \%$. Esses resultados confirmaram diferença estatística entre os grupos avaliados (Tabela 2).

A freqüência dos desconfortos músculoesqueléticos foi semelhante entre os grupos, na avaliação inicial. No final da gestação observouse que a maioria do GC passou a referir sintomas de desconfortos numa freqüência diária $(42,4 \%)$, enquanto que o GE se destacou pela freqüência quinzenal ou mais $(50,0 \%)$ dos sintomas (Tabela 3).

Os resultados do início do programa também não mostraram diferença significativa entre os grupos quanto a duração dos desconfortos músculo-esqueléticos. No final da gestação observou-se diferença significativa entre os grupos, com vantagem para o GE, em que 55,3\% das participantes referiram duração máxima de 1 hora. No GC, a duração ultrapassava as 3 horas em $69,7 \%$ das grávidas (Tabela 4).

Tabela 2 - Número (n) e porcentagem (\%) de gestantes classificadas de acordo com a intensidade dos sintomas de desconfortos músculo-esqueléticos no início e final do programa, nos grupos controle e estudo.

\begin{tabular}{|c|c|c|c|c|c|c|c|c|}
\hline \multirow{3}{*}{ Intensidade } & \multicolumn{4}{|c|}{ Controle } & \multicolumn{4}{|c|}{ Estudo } \\
\hline & \multicolumn{2}{|c|}{ Início* } & \multicolumn{2}{|c|}{ Final $^{* *}$} & \multicolumn{2}{|c|}{ Início* } & \multicolumn{2}{|c|}{ Final** } \\
\hline & $\mathrm{n}$ & $\%$ & $n$ & $\%$ & $\mathrm{n}$ & $\%$ & $n$ & $\%$ \\
\hline Sem sintomas & 12 & 36,4 & 4 & 12,1 & 6 & 15,8 & 9 & 23,7 \\
\hline Leve & 6 & 18,2 & - & - & 1 & 2,6 & 22 & 57,9 \\
\hline Moderada & 4 & 12,1 & - & - & 5 & 13,2 & 5 & 13,2 \\
\hline Grave & 7 & 18,4 & 20 & 60,6 & 14 & 36,8 & 2 & 5,3 \\
\hline Associada & 4 & 12,1 & 9 & 23,7 & 12 & 31,6 & 5 & 13,2 \\
\hline
\end{tabular}

${ }^{*} p=0,02$ - diferença significativa entre os grupos na avaliação inicial

${ }^{* *} p=0,00$ - diferença significativa entre os grupos na avaliação final. 
Tabela 3 - Percentual (\%) de gestantes distribuídas de acordo com a freqüência dos sintomas de desconfortos músculo-esqueléticos no início e final do programa, nos grupos controle e estudo.

\begin{tabular}{|c|c|c|c|c|c|c|c|c|}
\hline \multirow{3}{*}{ Frequência } & \multicolumn{4}{|c|}{ Controle } & \multicolumn{4}{|c|}{ Estudo } \\
\hline & \multicolumn{2}{|c|}{ Início* } & \multicolumn{2}{|c|}{ Final $^{* *}$} & \multicolumn{2}{|c|}{ Início* } & \multicolumn{2}{|c|}{ Final $^{\star *}$} \\
\hline & $\mathrm{n}$ & $\%$ & $\mathrm{n}$ & $\%$ & $\mathrm{n}$ & $\%$ & $\mathrm{n}$ & $\%$ \\
\hline Sem sintomas & 12 & 36,4 & 4 & 12,1 & 9 & 15,8 & 9 & 23,7 \\
\hline Quinzenal ou mais & 6 & 18,2 & - & - & 2 & 5,3 & 19 & 50,0 \\
\hline Semanal & 8 & 24,3 & 9 & 27,3 & 12 & 31,6 & 4 & 10,5 \\
\hline Diária & 3 & 9,1 & 14 & 42,4 & 6 & 15,8 & 1 & 2,6 \\
\hline Associada & 4 & 12,1 & 6 & 18,2 & 12 & 31,6 & 5 & 13,2 \\
\hline
\end{tabular}

* $p=0,05$ - diferença não significativa entre os grupos na avaliação inicial

${ }^{* *} p=0,00$ - diferença significativa entre os grupos na avaliação final.

Tabela 4 - Percentual (\%) de gestantes distribuídas de acordo com a duração dos sintomas de desconfortos músculo-esqueléticos no início e final do programa, nos grupos controle e estudo.

\begin{tabular}{|c|c|c|c|c|c|c|c|c|}
\hline \multirow{3}{*}{ Duração } & \multicolumn{4}{|c|}{ Controle } & \multicolumn{4}{|c|}{ Estudo } \\
\hline & \multicolumn{2}{|c|}{ Início* } & \multicolumn{2}{|c|}{ Final $^{* *}$} & \multicolumn{2}{|c|}{ Início* } & \multicolumn{2}{|c|}{ Final $^{* *}$} \\
\hline & $\mathrm{n}$ & $\%$ & $\mathrm{n}$ & $\%$ & $\mathrm{n}$ & $\%$ & $\mathrm{n}$ & $\%$ \\
\hline Sem sintomas & 12 & 36,4 & 4 & 12,1 & 6 & 15,8 & 9 & 23,7 \\
\hline Menor ou igual a 1 hora & 3 & 9,1 & - & - & 2 & 5,3 & 21 & 55,3 \\
\hline 2 a 3 horas & 7 & 21,2 & - & - & 6 & 15,8 & - & - \\
\hline Mais que 3 horas & 7 & 21,2 & 23 & 69,7 & 12 & 31,6 & 3 & 7,9 \\
\hline Associadas & 4 & 12,1 & 6 & 18,2 & 12 & 31,6 & 5 & 13,2 \\
\hline
\end{tabular}

${ }^{*} p=0,12$ - diferença não significativa entre os grupos na avaliação inicial

** $p=0,00$ - diferença significativa entre os grupos na avaliação final.

\section{Discussão}

Os resultados observados neste trabalho indicaram que os grupos foram homogêneos em relação à maioria das variáveis de controle. A equivalência entre características sociodemográficas dos sujeitos de uma determinada pesquisa foi considerada de importância por alguns autores, pelo risco de interferência na análise e comparação dos resultados ${ }^{3,6,22}$. Os IMC, avaliados no início do programa, foram comparáveis entre os grupos e confirmaram peso materno adequado ${ }^{19}$. Foi afastada, portanto, a possibilidade de qualquer interação indesejável entre atividade física e obesidade ou sobrepeso maternos. Nos dois grupos, independentemente da participação do PMPMa, a maioria das gestantes vivia com o companheiro e freqüentou a escola por, no máximo, 11 anos.

A idade materna foi a única variável não controlada na distribuição dos grupos de gestantes. A faixa etária do GE, além de mais jovem que o GC, caracterizou-o como exclusivamente composto de adolescentes. Embora a investigação não fosse direcionada para o binômio gravidez e ado- lescência, a população recrutada evidenciou alguns aspectos característicos deste tipo de gestação. A idade precoce pode ter favorecido a união conjugal antecipada e a interrupção dos estudos após o ensino fundamental. A mudança brusca e inesperada na vida destas gestantes, determinante de condições psicossociais inadequadas, aliada a características próprias do organismo em desenvolvimento, implicariam maior risco de complicações obstétricas e neonatais. Neste contexto, o preparo adequado para o parto e maternidade, oferecido para a totalidade das gestantes do GE, teve indicação precisa, no mínimo para a humanização da assistência ${ }^{3,4}$.

Merece destaque a presença de desconfortos músculo-esqueléticos na maioria das gestantes, tanto do GC como do GE. Observou-se, ainda, tendência desse grupo à maior proporção $(84,2 \%)$ de mulheres sintomáticas. A literatura afirma que a gestação é período propício para tais desconfortos $^{1,2,6,7,10,11}$.

No início do programa os sintomas de desconfortos localizavam-se, predominantemente, na região lombo-sacra, caracterizados por dor nos dois grupos de gestantes. A intensidade dos sintomas 
foi diferenciada, predominando a forma grave ou associada no GE, com freqüência mínima de uma semana. Este resultado pode ter contribuído para a tendência à maior proporção de gestantes sintomáticas no grupo a ser submetido à atividade fisioterápica. A constatação do maior acometimento da região lombo-sacra pode ser relacionada ao aumento do peso, à acentuação das curvaturas da coluna vertebral e à embebição gravídica, adaptações fisiológicas iniciadas com a gestação ${ }^{1,2,10,23}$. A repercussão desses sintomas, especialmente a diminuição da auto-estima e o comprometimento da sensação de "estar grávida", foi evidenciada por unanimidade entre vários autores ${ }^{2,4,6,10}$.

Apesar da não-diferenciação dos grupos quanto à presença dos sintomas de desconfortos músculo-esqueléticos no final da gestação, alguns efeitos desejáveis da prática da cinesioterapia foram destacados neste trabalho.

Associou-se à intervenção a menor proporção de gestantes sintomáticas e a redução da intensidade, freqüência e duração dos sintomas. Além disso, merece consideração a tendência do GE em apresentar maior proporção de gestantes sintomáticas no início e se equiparar ao GC no final da gestação. Estes resultados indicaram efeitos benéficos e validaram a cinesioterapia como recurso adjuvante e necessário ao atendimento pré-natal.

A literatura específica sobre desconfortos músculo-esqueléticos na gravidez é bastante escassa e não fornece referências para comparação dos resultados aqui observados. Os efeitos dos programas de preparação para o parto e maternidade, com atenção psicológica e física, continuam $\operatorname{controversos}^{24,25}$. As possiveis explicações são atribuídas, principalmente, à falta de padronização nos trabalhos, desde a aplicação e o desenvolvimento dos métodos até à análise dos resultados. Em estudo anterior, a análise de outros resultados da aplicação deste mesmo programa - o PMPMa -, confirmou que estas mesmas gestantes foram beneficiadas pelo controle da ansiedade no final da gestação e pela maior chance de parto vaginal $(83,8 \%)$, sem comprometimento do peso e da idade dos recém-nascidos. O índice de correlação de 0,30, específico entre ansiedade e via de parto, evidenciou que este não foi o único fator determinante desse resultado e que as outras atividades poderiam estar envolvidas ${ }^{4}$. Do mesmo modo, os efeitos da atividade fisioterápica podem ter ido além da redução das características dos sintomas músculo-esqueléticos. Isto reforça a validade de um programa multidisciplinar de preparo para o parto e maternidade.

Apesar de não influenciar na ocorrência dos desconfortos músculo-esqueléticos, as técnicas fisioterápicas aplicadas no PMPMa favoreceram a diminuição da intensidade, freqüência e duração e proporcionaram melhora na evolução dos sintomas. Embora haja a impossibilidade de individualizar as ações específicas de cada uma das atividades aplicadas no PMPMa, os resultados deste trabalho contribuíram para validar a relação direta entre a atividade fisioterápica e o controle dos sintomas de desconfortos músculo-esqueléticos na gestação. Portanto, essa prática se confirmou benéfica para assegurar a qualidade de vida materna na gestação.

\section{ABSTRACT}

Purpose: to study the effects of physiotherapeutic techniques applied by the Multidisciplinary Program of Preparation for the Childbirth and Maternity on musculoskeletal discomforts during pregnancy.

Methods: prospective cohort study, with 71 low-risk nulliparous women, distributed according to participation (study: $n=38$ ) or not (control; $n=33$ ). The Multidisciplinary Program of Preparation for Childbirh and Maternity had 10 meetings (18th to 38th week), with educational, physiotherapeutic, and interaction activities. Occurrence, characteristics, and evolution of musculoskeletal discomforts were compared by means of a specific questionnaire, both at the beginning and at the end of the program. The average of results of the initial assessment was compared through analysis of variance (ANOVA) followed by the F test. For the study between proportions in the beginning and at the end of the program the $\chi^{2}$ was used. The statistical significance was determined at $5 \%$ of limit $(p<0.05)$.

Results: at the beginning of the program, $63.6 \%$ of the pregnant women of the control group and $84.2 \%$ of the study group reported musculoskeletal symptoms $(p=0.05)$, characterized by back and posterior pelvic pain. In the control group, light intensity (18.2\%) and serious intensity pain (18.4) were predominant, while in the study group, the serious was $36.8 \%$, and the isolated or associated was 31.6\%. At the end, the control group showed symptoms of serious intensity (60.6\%), with daily frequency (42.4\%) and length of more than $3 \mathrm{~h}$ (69.7\%; $p<0.05)$. The study group reported light intensity (57.9\%) and bimonthly frequency (50.0\%) with a maximum length of one hour $(55.3 \%)(p<0.05)$. Symptom evolution was also differentiated and there were worsening in $63.6 \%$ of pregnant women of the control group and improvement in $65.8 \%$ of participants of the program $(p<0.05)$.

Conclusions: the physiotherapeutic techniques of the Multidisciplinary Program of Preparation for Childbirth and Maternity were related to a decrease in intensity, frequency and length and to a better evolution of musculoskeletal discomforts during pregnancy.

KEYWORDS: Normal pregnancy. Musculoskeletal discomforts. Maternity preparation program. 


\section{Referências}

1. Artal R, Wiswell RA, Drinkawater BL. O exercício na gravidez. 2a ed. São Paulo: Manole; 1999.

2. Polden M, Mantle J. Fisioterapia em ginecologia e obstetrícia. $2^{\mathrm{a}}$ ed. São Paulo: Ed. Santos; 2000.

3. Standing TS, el-Sabagh N, Brooten D. Maternal education during the perinatal period. Clin Perinatol 1998; 25:389-402.

4. Consonni HB. Repercussões de um programa multidisciplinar de preparo para o parto e maternidade - ansiedade materna e resultados perinatais [dissertação]. Botucatu: Univ. Estadual de São Paulo; 2001.

5. Sternfeld B. Physical activity and pregnancy outcome. Review and recommendations. Sports Med 1997; 23:33-47.

6. Koniak-Griffin D. Aerobic exercise, psychological well-being, and physical discomforts during adolescent pregnancy. Res Nurs Health 1994; 17:253-63.

7. Canto CEEM, Marques AP, Bio ER. A influência da atividade física materna sobre o curso gestacional e desenvolvimento fetal. Femina 1996; 25:459-65.

8. Génot C, Neiger H, Leroy A, Dufour M, Péniou G. Cinesioterapia: avaliações técnicas passivas e ativas do aparelho locomotor. Princípios. São Paulo: Editorial Médica Panamericana; 1989.

9. Colliton J. Back pain and pregnancy: Active management strategies. Phys Sportsmed 1996; 24:1-6.

10. Mauad Filho F, Araújo ACPF, Maranhão TMO, Casanova MS. Exercício físico na gravidez. Ginecol Obstet Atual 1999; 8:54-8.

11.Artal R, Sherman C. Exercise during pregnancy: Safe and beneficial for most. Phys Sportsmed 1999; 27:1-9.

12.De Vitta A. Bem-estar físico e saúde percebida: um estudo comparativo entre homens e mulheres adultos e idosos, sedentários e ativos [tese]. Campinas: Univ. Estadual de Campinas; 2001.

13. Courry HJ. Self-administered preventive programme for sedentary workers: reducing musculoskeletal symptoms or increasing awareness? Appl Ergon 1998; 29:415-21.

14.Vellay P. Parto sem dor. 5a ed. São Paulo: Ibrasa; 1984.

15.Dale B, Roeber J. Exercícios pré-natais: um guia para o bem-estar físico durante a gravidez. $1^{\text {a }}$ ed. São Paulo: Maltese-Forma; 1992.

16.Mens JM, Snijders CJ, Stam HJ. Diagonal trunk muscle exercises in peripartum pelvic pain: a randomized clinical trial. Phys Ther 2000; 80:116473.

17. Morais AAC, Tavares GM, Pezzin AC, Moana AA, Galväo HP, Faintuch J. Avaliação da composição corporal em gestantes de termo. Rev Assoc Med Bras 1997; 43:109-13.

18.SEADE. Pesquisa de condições de vida para a região metropolitana de São Paulo. São Paulo: SEADE; 1992.

19.Azeredo CAC. Fisioterapia respiratória moderna. $3^{\mathrm{a}}$ ed. São Paulo: Manole; 1999.

20.Cailliet R. Lombalgias. São Paulo: $1^{\text {a }}$ ed. São Paulo: Manole; 1979.

21.Fleiss JL. Statistical methods for rates and proportions. $2^{\text {nd }}$ ed. New York: Wiley; 1981. p.38-45.

22.Waldenstrom U. Experience of labor and birth in 1111 women. J Psychosom Res 1999; 47:471-82

23. Mesquita MRS, Bertini AM. Alterações do sistema osteoarticular na gravidez normal. In: FEBRASGO. Tratado de obstetrícia da Febrasgo. $1^{\text {a }}$ ed. Rio de Janeiro: Revinter; 2000. p.105-6.

24.Patton LL, English EC, Hambleton JD. Childbirth preparation and outcomes of labor and delivery in primiparous womem. J Fam Pract 1985; 20:375-8.

25.Heins HC Jr, Nance NW, McCarthy BJ, Efird CM. A randomized trial of nurse-midwifery prenatal care to reduce low birth weigth. Obstet Gynecol 1990; $75: 341-5$ 